\title{
Switch of substrate specificity of hyperthermophilic acylaminoacyl peptidase by combination of protein and solvent engineering
}

\author{
Chang Liu ${ }^{2,3^{*}}$, Guangyu Yang ${ }^{1 *}$, Lie $\mathrm{Wu}^{2}$, Guohe Tian $^{2}$, Zuoming Zhang ${ }^{2}$, Yan Feng ${ }^{1,2} \bowtie$ \\ ${ }^{1}$ State Key Laboratory of Microbial Metabolism, School of Life Sciences and Biotechnology, Shanghai Jiao Tong University, \\ Shanghai 200240, China \\ ${ }^{2}$ Key Laboratory for Molecular Enzymology and Engineering of Ministry of Education, Jilin University, Changchun 130023, China \\ ${ }^{3}$ Changchun Institute of Biological Products, China National Biotec Group, Changchun 130061, China \\ \ Correspondence: yfeng2009@sjtu.edu.cn \\ Received April 15, 2011 Accepted May 15, 2011
}

\section{ABSTRACT}

The inherent evolvability of promiscuous enzymes endows them with great potential to be artificially evolved for novel functions. Previously, we succeeded in transforming a promiscuous acylaminoacyl peptidase (apAAP) from the hyperthermophilic archaeon Aeropyrum pernix $\mathrm{K} 1$ into a specific carboxylesterase by making a single mutation. In order to fulfill the urgent requirement of thermostable lipolytic enzymes, in this paper we describe how the substrate preference of apAAP can be further changed from $p$-nitrophenyl caprylate ( $p N P-C 8)$ to $p$-nitrophenyl laurate ( $p N P-C 12)$ by protein and solvent engineering. After one round of directed evolution and subsequent saturation mutagenesis at selected residues in the active site, three variants with enhanced activity towards pNP-C12 were identified. Additionally, a combined mutant W474V/F488G/R526VI T560W was generated, which had the highest catalytic efficiency $\left(k_{\text {cat }} / K_{m}\right)$ for pNP-C12, about 71-fold higher than the wild type. Its activity was further increased by solvent engineering, resulting in an activity enhancement of 280 -fold compared with the wild type in the presence of $30 \%$ DMSO. The structural basis for the improved activity was studied by substrate docking and molecular dynamics simulation. It was revealed that $\mathrm{W} 474 \mathrm{~V}$ and F488G mutations caused a significant change in the geometry of the active center, which may facilitate binding and subsequent hydrolysis of bulky substrates. In conclusion, the combination of protein and solvent engineering may be an effective approach to improve the activities of promiscuous enzymes and could be used to create naturally rare hyperthermophilic enzymes.

KEYWORDS acylaminoacyl peptidase, esterase, substrate specificity, protein engineering, solvent engineering

\section{INTRODUCTION}

Lipolytic enzymes, such as lipases and esterases, catalyze a variety of reactions, including ester hydrolysis, esterification or transesterification, and they are important biocatalysts for the synthesis and resolution of enantio-pure drug precursors for the pharmaceutical industry (Houde et al., 2004; Verma et al., 2008). Because most substrates with long acyl chain lengths are hydrophobic and sparingly soluble in water, large amounts of organic solvents are usually added to the reaction system to increase substrate solubility and catalytic efficiency (Luetz et al., 2008). Unfortunately, most lipolytic enzymes easily lose their activities at high temperatures or in the presence of high concentrations of organic solvents. Consequently, there is considerable demand for novel lipolytic enzymes that are stable under these conditions.

Hyperthermophilic archaea, which grow at temperatures around $80^{\circ} \mathrm{C}-121^{\circ} \mathrm{C}$, are an important resource of thermostable enzymes (Niehaus et al., 1999; Imanaka and Atomi,

"These authors contributed equally to the work. 
2002). However, the lipid membranes of these heat-resistant organisms are unusual because they contain ether linkages instead of ester linkages between the glycerol backbone and the fatty acyl chains (Madigan and Martinko, 2006). Therefore, lipolytic enzymes that catalyze cleavage of ester bonds are not commonly found in such organisms. Lipases capable of utilizing water-insoluble substrates with long acyl chain lengths $(C \geqslant 10)$ are extremely rare in nature. Indeed, although many hyperthermophilic enzymes (optimal temperatures $>80^{\circ} \mathrm{C}$ ) have been isolated, very few lipases have been found in hyperthermophilic archaea, thus limiting their industrial applications (Salameh and Wiegel, 2007). Therefore, it is desirable to improve the catalytic character of natural enzymes to generate industrially useful lipases with high thermal stability and this can be achieved using artificial evolution.

Enzyme promiscuity, which is used to describe the ability of an enzyme to catalyze different chemical reactions from those for which it evolved, has received considerable attention over the last decade (O'Brien and Herschlag, 1999). It is widely accepted that promiscuous enzymes can evolve rapidly, allowing organisms to survive environmental changes. Recently, numerous reports have shown that promiscuous enzymes can evolve novel or altered functions, which provides a useful approach for generating new enzymes with specific properties (Aharoni et al., 2005; Hult and Berglund, 2007).

To investigate how promiscuous proteins evolve, we previously studied an acylaminoacyl peptidase from the hyperthermophilic archaeon Aeropyrum pernix K1 (apAAP) as a model enzyme. It is a typical promiscuous enzyme that exhibits peptidase and esterase activities (Gao et al., 2003). Moreover, it has an optimal temperature of $\sim 90^{\circ} \mathrm{C}$, and is extremely stable at high temperatures and in the presence of organic solvents (Gao et al., 2003). We previously solved the crystal structure of apAAP complexed with an organophosphorus substrate analog (Bartlam et al., 2004) and found that the enzyme is composed of two domains: a $\beta$ propeller domain and a canonical $\alpha / \beta$ hydrolase-fold domain. The latter is the catalytic domain, which includes the active site and a Ser445-Asp524-His556 catalytic triad. Moreover, we found a conserved residue in the active site (Arg526) which plays a crucial role in catalysis and substrate discrimination (Wang et al., 2006). Saturation mutagenesis at this position had dramatic effects on esterase and peptidase activities. Whereas the esterase activity of the wild type enzyme for $p$-nitrophenyl caprylate ( $p N P-C 8)$ was $\sim 7$ times higher than its peptidase activity for Ac-Leu-pnitroanilide, the esterase activities of mutants R526V and R526E were about 150- and 785-fold higher than their peptidase activities, respectively, using the same substrates (Wang et al., 2006). Further characterization showed that the mutants possessed hydrolytic activity towards a wide range of $p$-nitrophenyl alkanoate esters, with optimal acyl chain lengths ranging from $\mathrm{C} 4$ to $\mathrm{C} 8$. This substrate spectrum was similar to that of the wild type enzyme.

Because most industrially important substrates have much longer acyl chains ( $\mathrm{C} 12$ to $\mathrm{C} 18$ ) than those preferentially hydrolyzed by apAAP, we decided to modify the substrate specificity of apAAP from small substrates to bulky substrates with longer acyl chains. The R526V mutant generated in a previous study was shown to have high esterase activity, extreme thermal stability, and high tolerance to organic solvents, which makes it a good candidate for artificial evolution (Yang et al., 2009). In the present study, the esterase activity of the mutant R526V towards substrates with long acyl chains was enhanced by protein engineering and solvent optimization. After directed evolution and subsequent semi-rational protein design, several variants with increased activity compared to the parental enzyme were identified. Strikingly, some mutants displayed a preference for bulky substrates in the presence of high concentrations of organic solvents. To provide insights into the mechanisms by which the mutations exert their effects, site-directed mutagenesis, kinetic studies, substrate docking and molecular dynamics (MD) simulations were undertaken. The results suggest that hyperthermophilic promiscuous enzymes can be efficiently redesigned by the combination of protein and solvent engineering, which provides an alternative approach to obtaining novel enzymes to direct isolation from nature.

\section{RESULTS AND DISCUSSION}

\section{Protein engineering to increase the activity of apAAP}

In order to enhance the esterase activity of acylaminoacyl peptidase apAAP for long acyl chain substrates, the mutant R526V (named P01 in this study) was chosen as a starting point to be evolved. Given that the enzyme apAAP is a fairly large protein (581 residues) and most of the residues responsible for catalysis and substrate binding are located in the catalytic domain ( $\alpha / \beta$ hydrolase-fold domain), we only performed mutagenesis on the catalytic domain of mutant P01, generating a smaller but smarter random mutagenesis library. The DNA sequence encoding amino acids 320-581 of P01 was subjected to error-prone PCR under conditions that caused on average one or two amino acid substitutions per gene. The PCR products were cloned into a vector ( $\mathrm{pET}-15 \mathrm{~b}-$ APpropeller) that already contained DNA encoding the $\beta$ propeller domain to create a random mutagenesis library. A typical long acyl chain substrate, $p$-nitrophenyl laurate ( $p$ NP$\mathrm{C} 12$ ), was used to screen the mutant library. Acetonitrile (about 5\%) was added to the reaction system to increase the solubility of the substrate. After screening of $\sim 5000$ colonies from the random mutagenesis library of $\mathrm{P} 01$, a double mutant $\mathrm{R} 526 \mathrm{~V} / \mathrm{T} 560 \mathrm{~W}$ (E01) was identified with 1.5-fold increase in activity (Table 1). A second round of directed evolution was performed but no further improvement was found after 
Table 1 Specific activities of selected variants of apAAP with esterase, peptidase and thioesterase substrates

\begin{tabular}{llrrr}
\hline Enzyme & Mutations & $p N P-C 12$ & Ac-Leu-pNA & S-methyl thiobutanoate \\
\hline P01 $^{\mathrm{a}}$ & R526V & 775.19 & 1759.27 & 2909.18 \\
E01 $^{\mathrm{b}}$ & R526V/T560W & 1143.53 & 1519.40 & 2849.87 \\
S01 $^{\mathrm{c}}$ & F488G/R526V/T560W & 1781.20 & 21.57 & 189.52 \\
S02 $^{\mathrm{c}}$ & W474V/R526V/T560W & 3565.48 & 276.12 & 404.52 \\
C01 $^{\mathrm{d}}$ & W474V/F488G/R526V/T560W & 5554.91 & 22.75 & 751.06 \\
\hline
\end{tabular}

The activities were measured in $50 \mathrm{mmol} / \mathrm{L}$ phosphate buffer $(\mathrm{pH} 8.0)$ at $60^{\circ} \mathrm{C}$.

${ }^{a}$ Parental enzyme in this study.

${ }^{\mathrm{b}}$ Mutant from error-prone PCR library.

${ }^{c}$ Mutants from site-directed saturation mutagenesis libraries.

${ }^{d}$ Combined mutant.

screening of $\sim 10,000$ colonies.

It was reported that modifications at residues near the active site are more efficient than random mutagenesis for the improvement of enzyme properties (Morley and Kazlauskas, 2005; Paramesvaran et al., 2009). To speed up the process of enzyme evolution, residues within $5 \AA$ of the catalytic serine 445 (except for some essential residues involved in catalysis, such as the catalytic triad and the amino acids in the oxyanion hole) were chosen for saturation mutagenesis. As a result, eleven residues were selected as targets and saturation mutagenesis libraries based on the E01 mutant were constructed. About 300 clones from each library were screened to ensure that all 32 possible codons occurred with a probability greater than $99 \%$ (Rui et al., 2004). By analyzing the activity of the variants from these libraries, we found that mutations at position 446 resulted in a complete loss of activity, indicating that Tyr446 is an essential residue for catalysis. Some mutations of Trp474 and Phe488 increased catalysis of pNP-C12, whereas mutations of Pro370, Glu419, Tyr444, Tyr449, Ile489, Met477, Phe485 and Thr527 had little effect on activity or substrate specificity. After screening of $\sim 3000$ colonies, two positive mutants F488G/R526V/T560W (named S01) and W474V/R526V/ T560W (named S02) were selected from the libraries targeting at 488 and 474 sites, with increased activities of 1.55- and 3.11-fold compared with the mutant E01 (Table 1). The combined mutant W474V/F488G/R526V/T560W (named C01) was constructed by site-directed mutagenesis and contained all the mutations found to increase catalysis in this study (Table 1). The specific activity of $\mathrm{C} 01$ for $p$ NP-C12 was 7.16-fold higher than the mutant P01 and about 40 times higher than the wild type apAAP.

The specific activities of the selected mutants of apAAP with esterase, peptidase and thioesterase substrates were determined (Table 1). The mutations caused different effects on the three types of catalytic activities. Compared to the wild type, esterase activities of the mutants were increased significantly, but their peptidase and thioesterase activities were decreased (using Ac-Leu-pNA and S-methyl thiobutanoate as substrates, respectively). Compared with the parental enzyme P01, the mutants S01 and S02 had a
72.3- and 5.5-fold lower peptidase activity respectively than P01, and lower thioesterase activities by 19- and 7.0-fold, respectively. These results suggest that the residues at positions 488 and 474 of apAAP are important in determining substrate specificity.

\section{Solvent engineering to modify the substrate preference of the mutants}

Ester substrates with long acyl chains are usually insoluble in water. Therefore, organic solvents are usually added to the reaction system to increase the solubility of the substrate and promote catalysis. However, enzymatic activities are strongly affected by the solvent (Zaks and Klibanov, 1985; Stahl, et al., 1991). With a careful choice of the solvent system, it is possible to increase, decrease, or even reverse substrate preference and enantioselectivity (Tawaki and Klibanov, 1992; Wescott and Klibanov, 1993). Therefore, to maximize catalytic efficiency, we sought to optimize the solvent system for the mutants. Interestingly, we found that the catalytic efficiency of the mutants was significantly affected by polar solvents (Fig. 1). In the presence of organic solvents, the wild type apAAP, P01 and E01 mutants were strongly inhibited, whereas the activities of mutants S01, S02 and C01 were simulated under the same conditions. In the presence of $10 \%$ $(\mathrm{v} / \mathrm{v})$ of acetonitrile, the activity of wild type apAAP decreased by more than $70 \%$, but the mutants S01 and S02 showed increased activities by 1.7- and 3.2-fold, respectively. Similar effects were observed in the presence of DMSO and DMF. The activities of S01, S02 and C01 mutants were increased 2.5-5.63 times in the presence of 30\% DMSO, and 1.4-4.4 times in the presence of $20 \%$ DMF. Consequently, the activity enhancement of some mutants became even greater in the presence of high concentrations of organic solvents. In particular, the activity of the mutant C01 increased 240-fold compared with the wild type in the presence of $10 \%$ acetonitrile, 99-fold in $20 \%$ DMF and more than 280 -fold in $30 \%$ DMSO.

The substrate preference of the mutants was also affected by organic solvents. As shown in Fig. 2, wild type apAAP and mutants P01 and E01 maintained their substrate specificity 

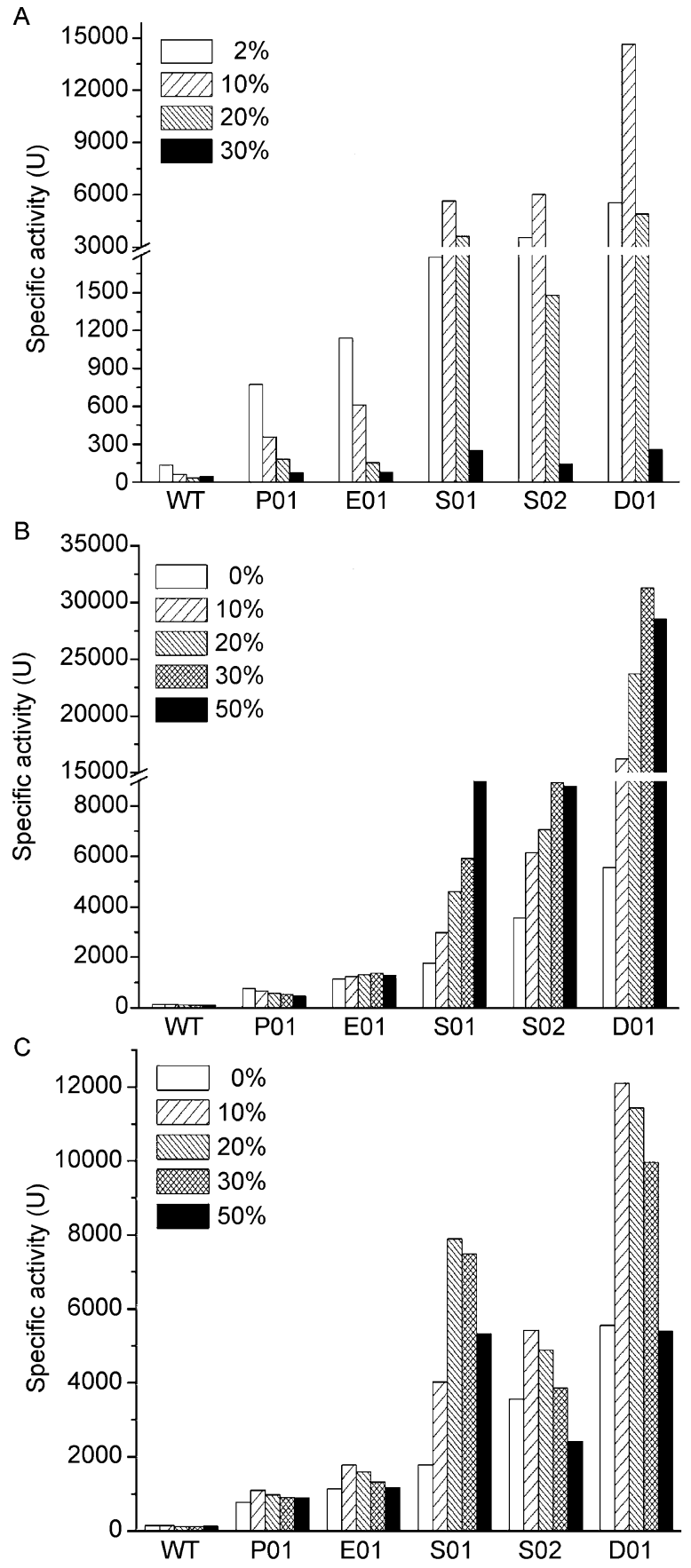

Figure 1. Specific activities of the variants of apAAP in different concentrations of acetonitrile (A), DMSO (B), and DMF (C) at $60^{\circ} \mathrm{C}$, with pNP-C12 as the substrate.

towards esters with short to medium acyl chain lengths (C4 to C8). Conversely, the mutants S01, S02 and C01 favored longchain substrates in the presence of high concentrations of organic solvents. In the presence of $20 \%$ DMF, the specific activities of wild type apAAP and the mutants P01 and E01 for the substrate $p$ NP-C 12 were about $27 \%, 36 \%$ and $46 \%$ of that for $p$-nitrophenyl butyrate (pNP-C4), respectively. However, mutants $\mathrm{S} 01, \mathrm{~S} 02$ and $\mathrm{C} 01$ hydrolyzed $p \mathrm{NP}-\mathrm{C} 12$ at a rate 5.5, 1.8 and 8.2 times faster than pNP-C4, respectively. The activities of these mutants towards other long chain $(C>10)$ esters were also substantially increased in the presence of organic solvents. As shown in Fig. $2 \mathrm{E}$, the ratio of the activities for $p N P-C 12$ and $p N P-C 4$ increased 12-, 18.3- and 19 -fold for the mutant $\mathrm{C} 01$ in the presence of $10 \%$ acetonitrile, $30 \%$ DMSO and $20 \%$ DMF, respectively.

\section{Kinetic and structural analyses of the mutants}

To investigate the effects of the mutations on the catalytic mechanism, additional mutants with diverse side chains at positions 474 and 488 were constructed and subjected to kinetic analysis alongside previously selected mutants (Table 2). The results revealed that the enhanced activity of E01 was due to a significant decrease in $K_{m}$ rather than an increase in $k_{\text {cat }}$, suggesting that the mutation increased the binding efficiency of the substrate. In contrast, extra mutations in S01, S02 and C01 at positions 474 and 488 resulted in an increase in $k_{\text {cat }}$. Interestingly, it seems that the hydrophobicity of the side chain at position 488 directly affects the value of $k_{\text {cat }}$. When the size of the side chain at position 488 was systematically decreased from Trp to Gly, the corresponding $k_{\text {cat }}$ values increased by about 10 times, indicating a negative correlation between $k_{\text {cat }}$ and the size of the side chain. Since position 488 is close to the active-site residue serine 445 (within $5 \AA$ ), we propose that a small side chain at position 488 may allow the reaction intermediate to adopt a more favorable orientation, decreasing the free energy barrier of the reaction and enhancing catalytic efficiency. Similarly, mutations at position 474 showed that small neutral side chains increased catalysis (increasing $k_{\text {cat }}$ ) as well as substrate binding (decreasing $K_{\mathrm{m}}$ ). C01 had the highest esterase activity of all the mutants investigated in this study. Compared with the wild type enzyme, C01 had a 19-fold higher $k_{\text {cat }}$ value and a 3.7-fold lower $K_{\mathrm{m}}$ value, which resulted in a 71.4-fold rise in $k_{\text {cat }} / K_{\mathrm{m}}$. We also attempted to measure kinetic parameters in the presence of high concentrations of organic solvents. Under these conditions, the data no longer fitted the Michaelis-Menten equation, suggesting that the catalytic behavior was considerably different when the solvents were present.

To understand the structure-function relationship of the mutants, the structure of $\mathrm{C} 01$ was modeled and docked with the substrate pNP-C12 (Fig. 3A). Analysis of the structure revealed that the distance between the $\mathrm{C}_{\alpha}$ of Trp560 and the catalytic residue Ser445 is $14.6 \AA$, suggesting that the facilitation of substrate binding caused by the T560W mutation was due to long-range interactions. In contrast, residues 474 and 488 are in the vicinity of the active center. Trp474 is located at the bottom of the substrate binding 

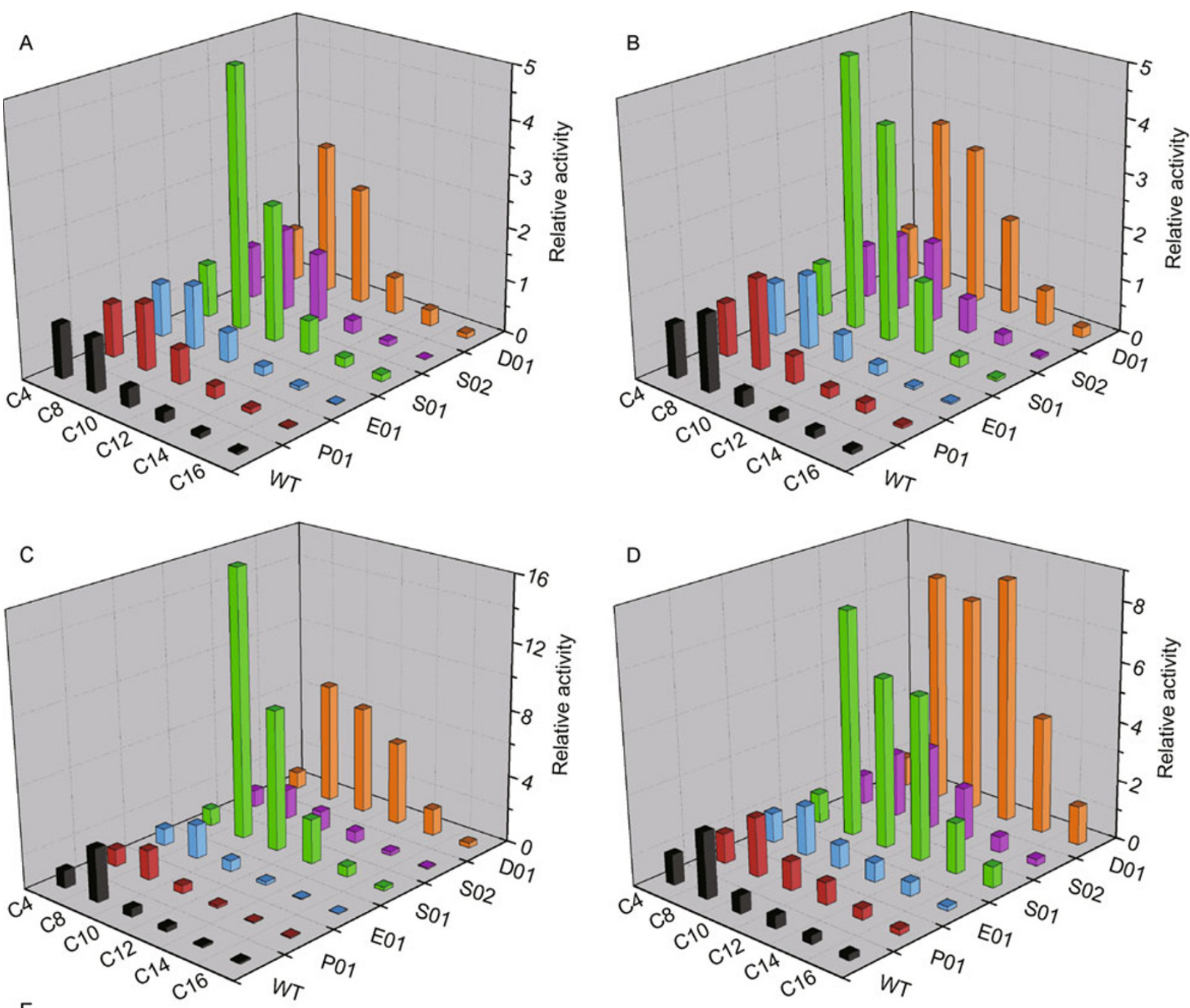

$\mathrm{E}$

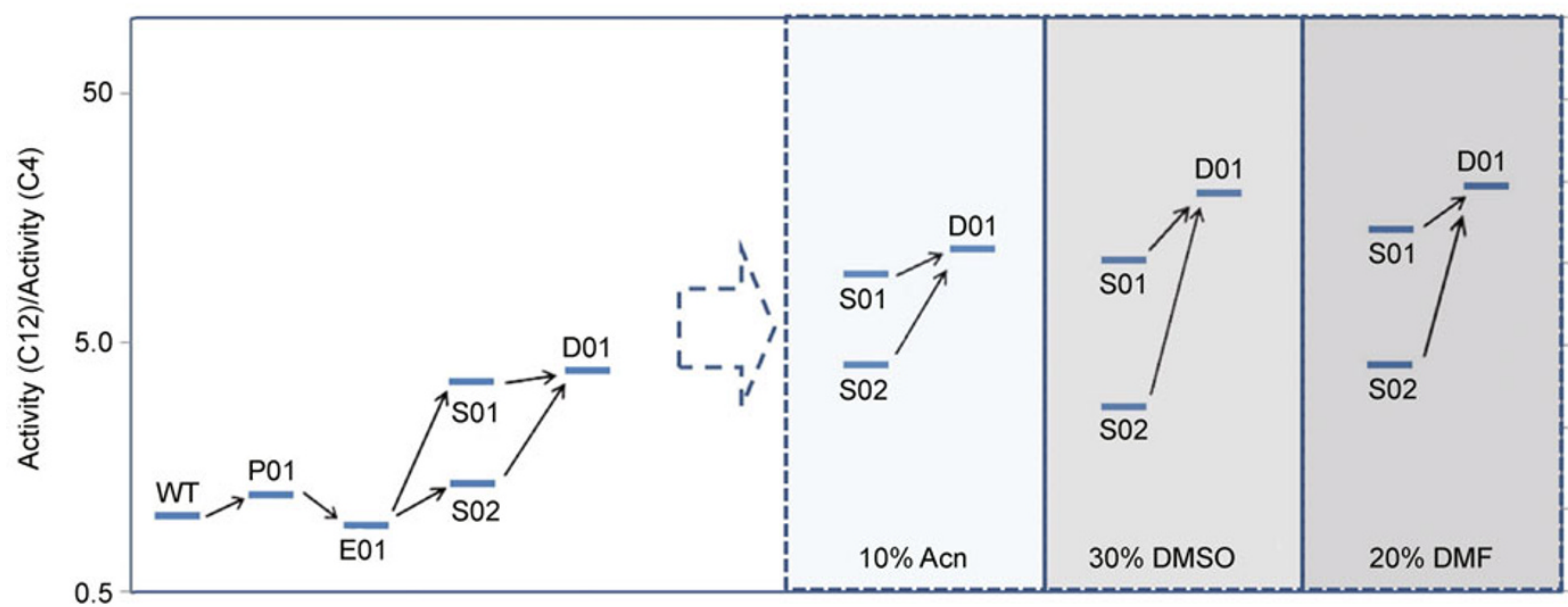

Figure 2. Substrate specificities of the variants of apAAP towards p-nitrophenyl alkanoate esters with various acyl chain lengths. (A) Specificities in the absence of organic solvents. (B) Specificities in the presence of $10 \%$ acetonitrile. (C) Specificities in the presence of $30 \%$ DMSO. (D) Specificities in the presence of $20 \%$ DMF. (E) Evolution pathway of the substrate selectivity between $p$ NP-C12 and $p$ NP-C4. Values for wild type apAAP were assigned as one. 
Table 2 Kinetic parameters of wild type apAAP and its mutants for the hydrolysis of $p$ NP-C 12 at $60^{\circ} \mathrm{C}$

\begin{tabular}{llccc}
\hline Enzyme & Mutant & $k_{\text {cat }}\left(\mathrm{s}^{-1}\right)$ & $K_{m}(\mu \mathrm{mol} / \mathrm{L})$ & $k_{\text {cat }} / K_{m}\left(\mathrm{~s}^{-1} / \mathrm{mmol}^{-\mathrm{L}^{-1}}\right)$ \\
\hline WT & Wild type & 1.35 & 8.62 & 157.2 \\
P01 & R526V & 5.47 & 11.8 & 462.5 \\
E01 & R526V/T560W & 7.96 & 1.22 & 6528 \\
S01 & F488G/R526V/T560W & 17.17 & 8.34 & 2059 \\
S011 & F488A/R526V/T560W & 12.08 & 7.37 & 1638 \\
S012 & F488S/R526V/T560W & 10.45 & 5.79 & 1804 \\
S013 & F488P/R526V/T560W & 8.17 & 3.88 & 2107 \\
S014 & F488Y/R526V/T560W & 2.05 & 0.76 & 2703 \\
S015 & F488W/R526V/T560W & 1.73 & 1.01 & 1712 \\
S02 & W474A/R526V/T560W & 12.61 & 1.83 & 6876 \\
S021 & W474Q/R526V/T560W & 5.29 & 0.75 & 7018 \\
S022 & W474V/R526V/T560W & 23.50 & 2.43 & 9662 \\
C01 & W474V/F488G/R526V/T560W & 26.25 & 2.34 & 11218 \\
\hline
\end{tabular}

The kinetic parameters were determined according to Lineweaver-Burk double reciprocal plots. Data are reported as means of at least two experiments.
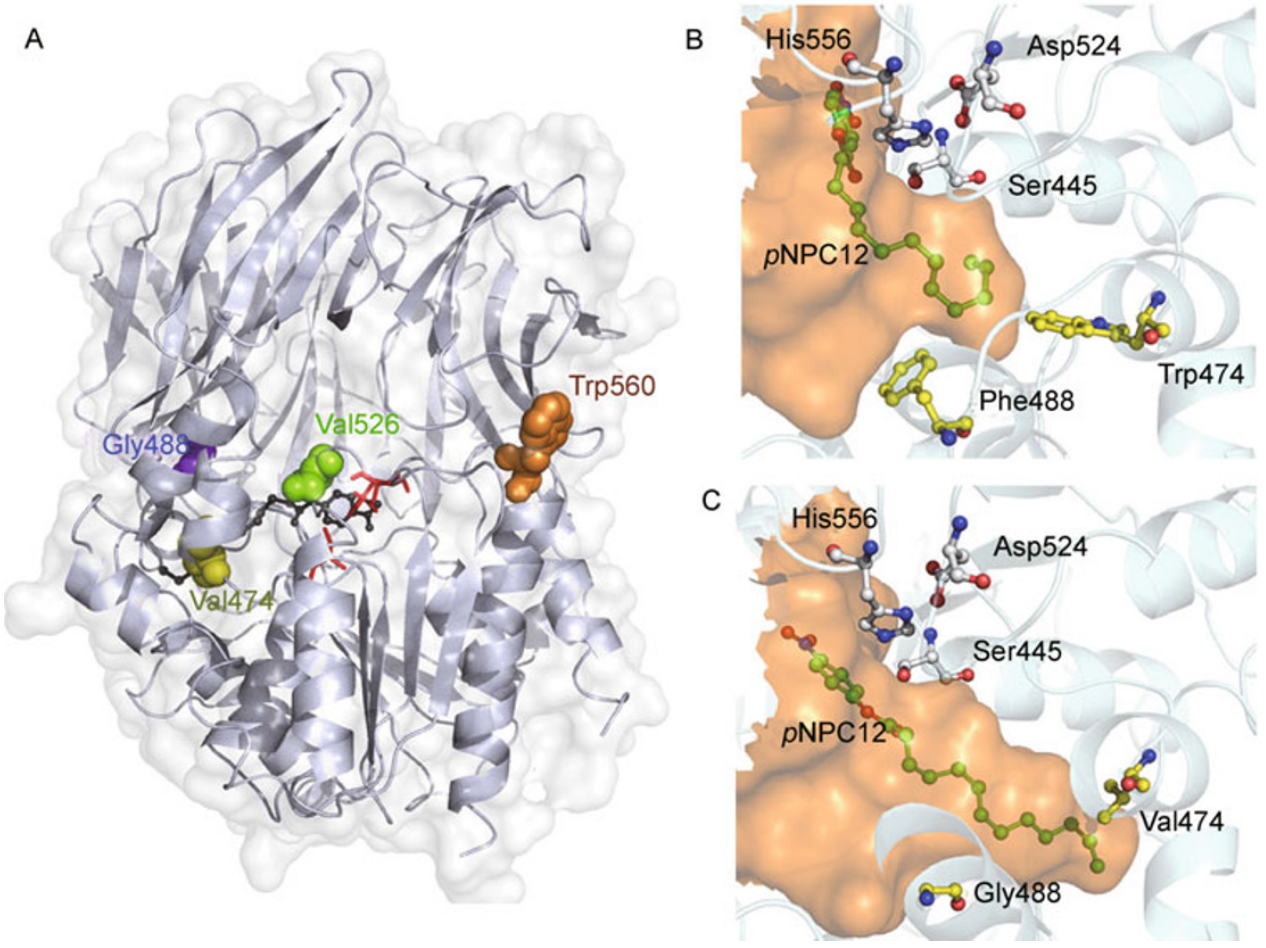

Figure 3. Modeled structures of selected variants of apAAP. (A) The overall structure of mutant $\mathrm{C} 01$ identified in this study. The main chain of the enzyme is shown as a ribbon, while the mutation sites are shown as spheres. The catalytic triad of the enzyme is shown in red, and the substrate pNP-C12 is shown in dark grey. (B) Molecular geometry of the substrate binding pocket of the parental enzyme P01. (C) Molecular geometry of the substrate binding pocket of the best mutant $\mathrm{C} 01$.

pocket, with its bulky side chain forming the end of the substrate binding tunnel. In contrast, Phe488 is located in the lateral wall of the substrate binding pocket. After molecular docking, the model of the pNP-C12/P01 complex showed that the side chain of Trp474 prevents the long acyl chain of
pNP-C12 from extending, while the side chain of Phe488 restricts the orientation of the substrate (Fig. 3B). As a result, $p N P-C 12$ lies in a twisted conformation inside the substrate binding pocket, which is unfavorable for both substrate binding and catalysis. However, the side chains of Val474 
and Gly488 in the model of the pNP-C12/C01 complex (Fig. $3 \mathrm{C}$ ) are much smaller than the corresponding residues in the wild type, resulting in a wider $(9.8 \AA$ vs $8.3 \AA$ ) and deeper (16.8 $\AA$ vs $7.8 \AA$ ) substrate binding pocket. Consequently, the volume of the substrate binding pocket (calculated using CASTp http://sts.bioengr.uic.edu/castp/) is significantly larger in the pNP-C12/C01 complex (502.9 $\AA^{3}$ vs $148.7 \AA^{3}$ in the wild type), which facilitates the binding of substrates with long acyl chains. This result suggests that the increased catalytic activity of mutant $\mathrm{C} 01$ towards long chain substrates is due to changes in the active site brought about by protein engineering.

\section{Conformation analysis of the mutants by MD simulations}

The effect of organic solvents on protein structure is difficult to assess by crystal structure determination or NMR analysis, yet MD simulations have been shown to be a powerful tool for investigating the behavior of enzymes in the presence of organic solvents (Soares et al., 2003; Yang et al., 2004). To gain an insight into the underlying mechanism of the solvent effect, MD simulations were run for the mutants S01, S02 and their parental enzyme P01, mimicking the condition when $10 \%$ acetonitrile was present. The root-mean-square deviation (RMSD) of the backbone atoms stabilized after $1 \mathrm{~ns}$ of simulation and data for subsequent 1 ns were collected for further analysis (Fig. 4A). The results showed that the secondary structures of all the protein molecules were generally stable, since more than $90 \%$ of the $\alpha$-helix and $\beta$ sheet were preserved (Fig.4B). In addition, the conformation and key interactions in the active site were also stable during the simulation period, e.g. the hydrogen bonding between Ser445 $\mathrm{O}_{Y}$ and His556 $\mathrm{N}_{\varepsilon 2}$, and between the carboxyl oxygen of Asp524 and His556 $\mathrm{N}_{\varepsilon 1}$, were preserved well (Fig. 4C).
A

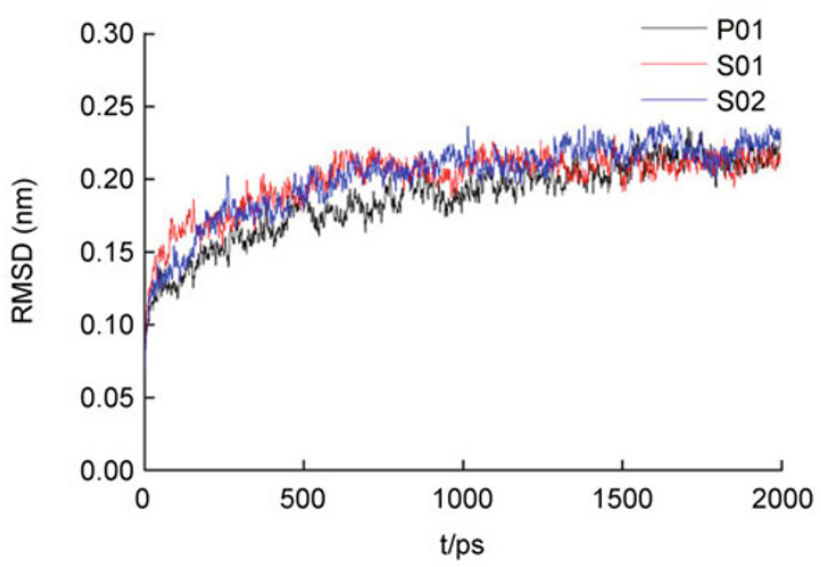

C

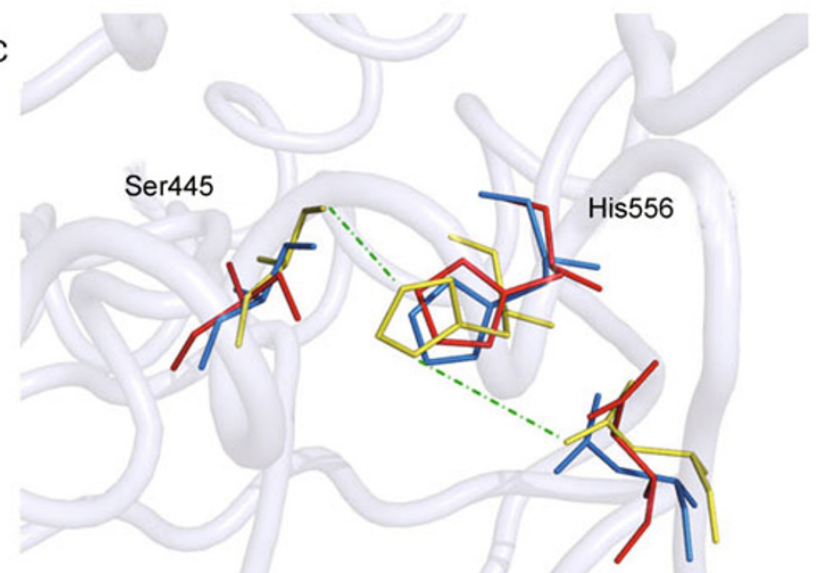

Asp 524
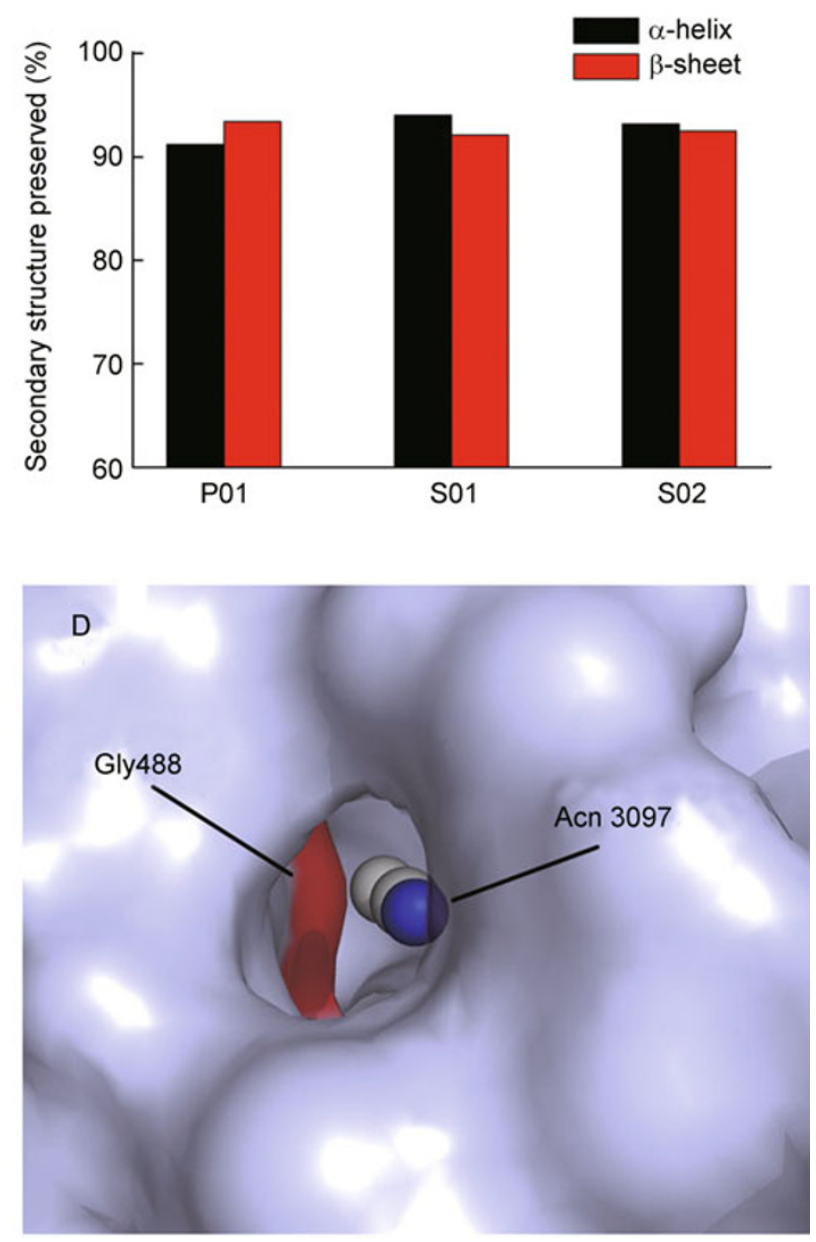

Figure 4. Results of molecular dynamics simulations of the variants of apAAP. (A) RMSD of the backbone atoms of P01 (black), S01 (red), and S02 (blue) as a function of time. (B) The percentage of preserved secondary structure of the mutants after the MD simulation reached equilibrium. (C) Superimposition of the active sites of the mutants P01 (yellow), S01 (red), and S02 (blue) after the MD simulation reached equilibrium. The backbone and hydrogen bonding networks represented in the figure are belonging to P01. (D) A snapshot of the penetration of acetonitrile 3097 into the S01 mutant. 
Detailed analyses revealed that several acetonitrile molecules penetrated the enzymes. The acetonitrile penetration for mutants S01 and S02 was more pronounced than for P01; three acetonitrile molecules were found in the interior of S01 and S02, whereas only one was found in P01 at the end of the MD simulations. These results may indicate that $\mathrm{S} 01$ and $\mathrm{S} 02$ have a higher conformational flexibility than P01, allowing acetonitrile molecules to penetrate. Thus, since the catalytic machinery of the enzymes was not disrupted by the penetration of acetonitrile molecules, the higher activities of S01 and S02 in the presence of organic solvents may be attributed to the fact that they have a more dynamic structure than P01. Interestingly, acetonitrile molecule 3097 (Acn 3097) penetrated S01 in the region where residue 488 was located (Fig. 4D). It might also have caused the slight enlargement of the substrate binding pocket observed in this mutant. Acetonitrile may cause substrates to gather near the active site at a high concentration and thus promote the reaction by an "induced fit" mechanism due to the flexible conformation of the mutant. These results suggest that the combination of site-directed mutagenesis and solvent engineering can be used to optimize the microenvironment of apAAP and enhance catalysis.

\section{CONCLUSION}

In this work, we successfully evolved a hyperthermophilic acylaminoacyl peptidase (apAAP) by protein and solvent engineering to enhance its activity towards ester substrates with long acyl chains. The mutant C01 retained high thermostability and had the highest esterase activity, with an activity for $p$ NP-C12 of up to 280 -fold that of the wild type. In addition, the substrate preference of $\mathrm{C} 01$ switched from substrates with short chains to substrates with longer chains in the presence of organic solvents, with the preferred substrate changing from $p N P-C 8$ to $p N P-C 12$. Kinetic analysis and substrate docking provided insights into the mechanism of the mutation effects: the mutations at positions 474 and 488 caused significant changes in the geometry of the active site, which presumably facilitated the binding and hydrolysis of bulky substrates. Molecular dynamics simulations indicated that acetonitrile in the environment and its penetration into the active site may cause changes in the microenvironment of the active site which enhance catalysis. Together, this work demonstrates that the combination of protein and solvent engineering is a powerful approach for redesigning hyperthermophilic promiscuous enzymes to generate novel enzymes with potential industrial applications.

\section{MATERIALS AND METHODS}

\section{Construction of random mutagenesis libraries}

DNA encoding the mutant R526V of apAAP was used as a template for error-prone PCR according to established protocols (Vartanian et al., 2001). Briefly, 1-10 ng of pET15b-apAAP_R526V plasmid was amplified by PCR containing a 1:5 ratio of AG:TC dNTPs supplemented with $6 \mathrm{mmol} / \mathrm{L} \mathrm{MgCl}_{2}$, using oligonucleotides apAAP-f (TTGGAGGCCCCTCCCGGCGGTGGATCCCTATCTCCT, Ncol restriction site underlined) and apAAP-r (CCAGGGTAACCATGGGAGGGTGGTTC, BamHI restriction site underlined) as primers. The PCR program was as follows: $1 \mathrm{~min}$ at $94^{\circ} \mathrm{C}$, followed by 30 cycles of $1 \mathrm{~min}$ at $94^{\circ} \mathrm{C}, 45 \mathrm{~s}$ at $45^{\circ} \mathrm{C}$, and $1 \mathrm{~min}$ at $72^{\circ} \mathrm{C}$. Two units of $D p n$ I were then added to the PCR solution and incubated at $37^{\circ} \mathrm{C}$ for $1 \mathrm{~h}$ to eliminate the template DNA. The mutated genes were digested by $\mathrm{Ncol}$ and $\mathrm{BamHI}$ and then ligated into the $\mathrm{pET} 15 \mathrm{~b}$ vector. The ligation mixture was used to transform E. coli BL21-CodonPlus (DE3)-RIL competent cells by electroporation. The transformed cells were grown overnight on 2YT agar plates supplemented with ampicillin $(100 \mu \mathrm{g} / \mathrm{mL})$ at $37^{\circ} \mathrm{C}$. The resulting colonies were then picked into 96-well plates and grown overnight. The plates were duplicated and protein expression was induced by $1 \mathrm{mmol} / \mathrm{L}$ isopropyl 1 -thio- $\beta-D$ galactopyranoside (IPTG). After $6 \mathrm{~h}$ of induction at $37^{\circ} \mathrm{C}$, cells were harvested by centrifugation and screened.

\section{Construction of site-directed saturation mutagenesis libraries}

The codons for target residues in the substrate binding pocket of apAAP were randomized by QuickChange site-directed mutagenesis according to the protocol of Zheng et al., (2004). The degenerate primers are listed in Table S1. The PCR program was as follows: 16 cycles at $94^{\circ} \mathrm{C}$ for $1 \mathrm{~min}, 60^{\circ} \mathrm{C}$ for $1 \mathrm{~min}$, and $68^{\circ} \mathrm{C}$ for $7 \mathrm{~min}$. After reaction, the PCR mixture was incubated at $72^{\circ} \mathrm{C}$ for a further $20 \mathrm{~min}$ and then stored at $4^{\circ} \mathrm{C}$. The library was subsequently generated by purification, self-ligation, and transformation into E. coli BL21CodonPlus (DE3)-RIL strain as described in the protocol.

\section{Screening of the libraries}

Harvested E. coli cells were frozen and thawed three times to release the expressed enzyme. Cell pellets were resuspended in $200 \mu \mathrm{L}$ of $50 \mathrm{mmol} / \mathrm{L}$ phosphate buffer ( $\mathrm{pH} \mathrm{8.0)}$ ), and the turbidity of each well was measured at $600 \mathrm{~nm}$ by a 96 -well plate reader (Thermo labsystems, Franklin, MA). The crude bacterial extracts were then incubated at $80^{\circ} \mathrm{C}$ for $30 \mathrm{~min}$ and subjected to centrifugation to remove heat-induced aggregates of proteins. A $100 \mu \mathrm{L}$ aliquot from each well was pipetted into a new 96-well plate, to which $100 \mu \mathrm{L}$ of substrate solution containing $0.2 \mathrm{mmol} / \mathrm{L} p N P-C 12$ in $50 \mathrm{mmol} / \mathrm{L}$ phosphate buffer $(\mathrm{pH}$ 8.0) was added. The plate was incubated at $60^{\circ} \mathrm{C}$ for $20 \mathrm{~min}$ and esterase activity in each assay well was determined by measuring the absorbance at $405 \mathrm{~nm}$. The change in $A_{405}\left(\Delta A_{405}\right)$ of each well was normalized by the corresponding $A_{600}$, and the ratio $r=\Delta A_{405} / A_{600}$ was used to estimate the activity of each colony.

\section{Protein expression, purification and enzymatic assays}

Variants of apAAP were expressed in E. coli BL21-CodonPlus (DE3)RIL and purified as described previously (Wang et al., 2006). Esterase, thioesterase, and peptidase activities were determined according to previously described protocols, using pNP-C12, Smethyl thiobutanoate, and Ac-Leu- $p$-nitroanilide as substrates, respectively (Mandrich et al., 2006; Yang et al., 2009). The standard 
enzymatic assay was performed in $50 \mathrm{mmol} / \mathrm{L}$ phosphate buffer $(\mathrm{pH}$ 8.0 ) at $60^{\circ} \mathrm{C}$. One active unit is defined as the amount of enzyme that liberates $1 \mu \mathrm{mol}$ of product per minute. Protein concentration was determined according to the Bradford method, and bovine serum albumin was used as a standard. The activity data were reported as mean values of at least triplicate measurements.

\section{Activity and substrate specificity in aqueous-organic media}

The activity of variants of apAAP was measured in the standard reaction system with acetonitrile, DMF or DMSO added to final concentrations of $2 \%-50 \%(\mathrm{~V} / \mathrm{V})$. The substrate selectivity of the variants in organic solvents against various $p N P$ esters with acyl chain length ranging from $\mathrm{C} 4$ to $\mathrm{C} 16$ was determined in the presence of $0-50 \%$ acetonitrile, DMF or DMSO.

\section{Steady-state kinetics}

The Michaelis-Menten parameters $K_{\mathrm{m}}$ and $k_{\text {cat }}$ of the variants were determined in $50 \mathrm{mmol} / \mathrm{L}$ phosphate buffer $(\mathrm{pH} 8.0)$ at $60^{\circ} \mathrm{C}$, using $p N P-C 12$ as substrate. The initial steady-state velocities of substrate hydrolysis were monitored with 7-9 concentrations of substrate ranging from 20 to $200 \mu \mathrm{mol} / \mathrm{L}$. All kinetic data were analyzed by linear regression to a Lineweaver-Burk double reciprocal plot.

\section{Molecular modeling and substrate docking}

Molecular modeling of the apAAP mutants and substrate docking were performed according to a previously described method (Wang et al., 2006), except that $p$ NP-C12 was used as a ligand instead of $p$ NPC8. Briefly, mutations were introduced by MODELLER 9v7 (MartiRenom et al., 2000), based on a $1.8 \AA$ crystallographic structure of wild type apAAP (PDB: 1VE6) and scored by PROCHECK (Laskowski et al., 1993) and Profile3D (Luthy et al., 1992) in the Insight II software package (Accelrys Inc., San Diego, CA). The advanced docking program Affinity was used to perform the automated molecular docking. The docked complex structures were selected based on interaction energy and geometrical matching quality. For example, the distance between the $O_{Y}$ of Ser in the catalytic triad and the carbonyl carbon of the substrate should be within $4 \AA$, and the distance between the oxyanion hole in the enzymes and the carbonyl carbon of the substrate should be within 5 $\AA$. The substrate binding pocket was analyzed using the program HOLLOW (Ho and Gruswitz, 2008). The structures of the mutants were analyzed by pyMOL software (Schrödinger, Portland, OR).

\section{Molecular dynamics simulation}

The MD simulations were performed with periodic boundary conditions using the GROMACS 4.5 software package (Van Der Spoel et al., 2005). About 900 acetonitrile molecules were added to a box containing an apAAP protein molecule and about 23,000 water molecules, using PACKMOL software (Martínez et al., 2009), to simulate the presence of $10 \%(\mathrm{v} / \mathrm{v})$ organic solvent in the system. The force field of acetonitrile was generated by PRODRG (Schuettelkopf and van Aalten, 2004). Each structure was energy-minimized by applying up to 500 steps using the steepest descent method until the results converged to within $100 \mathrm{kcal} \cdot \mathrm{mol}^{-1} \cdot \AA^{-1}$. A short MD simulation was performed starting from the energy-minimized structures for
$50 \mathrm{ps}$ at $333 \mathrm{~K}$ to allow equilibration. The actual simulation to explore conformational space was performed for $2 \mathrm{~ns}$ at the same temperature (time step $=2 \mathrm{fs}$ ). Snapshots were taken every $1 \mathrm{ps}$, to generate 2001 different conformers. The trajectories obtained for each mutant were analyzed.

\section{ACKNOWLEDGEMENTS}

We acknowledge Dr. Danni Liu for helpful discussions about the manuscript. This work was supported by the National Basic Research Program of China (973 Program), and the National Natural Science Foundation of China.

\section{ABBREVIATIONS}

apAAP, acylaminoacyl peptidase from Aeropyrum pernix K1; IPTG, isopropyl-thiogalactopyranoside; $p N P-C 4, p$-nitrophenyl butyrate; pNP-C8, $p$-nitrophenyl caprylate; pNP-C12, $p$-nitrophenyllaurate; Ac-Leu- $p N A$, Ac-Leu- $p$-nitroanilide; DMF, dimethylformamide; DMSO, dimethyl sulfoxide; MD, molecular dynamics

Supplementary material is available in the online version of this article at http://dx.doi.org/10.1007/s13238-011-1057-7 and is accessible for authorized users.

\section{REFERENCES}

Aharoni, A., Gaidukov, L., Khersonsky, O., Mc, Q.G.S., Roodveldt, C., and Tawfik, D.S. (2005). The 'evolvability' of promiscuous protein functions. Nat Genet 37, 73-76.

Bartlam, M., Wang, G., Yang, H., Gao, R., Zhao, X., Xie, G., Cao, S., Feng, Y., and Rao, Z. (2004). Crystal structure of an acylpeptide hydrolase/esterase from Aeropyrum pernix K1. Structure 12, 1481-1488.

Gao, R., Feng, Y., Ishikawa, K., Ishida, H., Ando, S., Kosugi, Y., and Cao, S. (2003). Cloning, purification and properties of a hyperthermophilic esterase from archaeon Aeropyrum pernix K1. J Mol Catal, B Enzym 24-25, 1-8.

Ho, B.K., and Gruswitz, F. (2008). HOLLOW: generating accurate representations of channel and interior surfaces in molecular structures. BMC Struct Biol 8, 49.

Houde, A., Kademi, A., and Leblanc, D. (2004). Lipases and their industrial applications: an overview. Appl Biochem Biotechnol 118, 155-170.

Hult, K., and Berglund, P. (2007). Enzyme promiscuity: mechanism and applications. Trends Biotechnol 25, 231-238.

Imanaka, T., and Atomi, H. (2002). Catalyzing "hot" reactions: enzymes from hyperthermophilic Archaea. Chem Rec 2, 149-163.

Laskowski, R.A., MacArthur, M.W., Moss, D.S., and Thornton, J.M. (1993). PROCHECK: a program to check the stereochemical quality of protein structures. J Appl Cryst 26, 283-291.

Luetz, S., Giver, L., and Lalonde, J. (2008). Engineered enzymes for chemical production. Biotechnol Bioeng 101, 647-653.

Luthy, R., Bowie, J.U., and Eisenberg, D. (1992). Assessment of protein models with three-dimensional profiles. Nature 356, 83-85.

Madigan, M.T., and Martinko, J.M. (2006). Brock Biology of Microorganisms, 11th ed. Beijing: Science Press.

Mandrich, L., Manco, G., Rossi, M., Floris, E., Jansen-van den Bosch, T., Smit, G., and Wouters, J.A. (2006). Alicyclobacillus acidocal- 
darius thermophilic esterase EST2's activity in milk and cheese models. Appl Environ Microbiol 72, 3191-3197.

Marti-Renom, M.A., Stuart, A.C., Fiser, A., Sanchez, R., Melo, F., and Sali, A. (2000). Comparative protein structure modeling of genes and genomes. Annu Rev Biophys Biomol Struct 29, 291-325.

Martínez, L., Andrade, R., Birgin, E.G., and Martinez, J.M. (2009). PACKMOL: a package for building initial configurations for molecular dynamics simulations. J Comput Chem 30, 2157-2164.

Morley, K.L., and Kazlauskas, R.J. (2005). Improving enzyme properties: when are closer mutations better? Trends Biotechnol 23, 231-237.

Niehaus, F., Bertoldo, C., Kahler, M., and Antranikian, G. (1999). Extremophiles as a source of novel enzymes for industrial application. Appl Microbiol Biotechnol 51, 711-729.

O'Brien, P.J., and Herschlag, D. (1999). Catalytic promiscuity and the evolution of new enzymatic activities. Chem Biol 6, R91-R105.

Paramesvaran, J., Hibbert, E.G., Russell, A.J., and Dalby, P.A. (2009). Distributions of enzyme residues yielding mutants with improved substrate specificities from two different directed evolution strategies. Protein Eng Des Sel 22, 401-411.

Rui, L., Kwon, Y.M., Fishman, A., Reardon, K.F., and Wood, T.K. (2004). Saturation mutagenesis of toluene ortho-monooxygenase of Burkholderia cepacia G4 for Enhanced 1-naphthol synthesis and chloroform degradation. Appl Environ Microbiol 70, 3246-3252.

Salameh, M., and Wiegel, J. (2007). Lipases from extremophiles and potential for industrial applications. Adv Appl Microbiol 61, 253-283.

Schuttelkopf, A.W., and van Aalten, D.M. (2004). PRODRG: a tool for high-throughput crystallography of protein-ligand complexes. Acta Crystallogr D Biol Crystallogr 60, 1355-1363.

Soares, C.M., Teixeira, V.H., and Baptista, A.M. (2003). Protein structure and dynamics in nonaqueous solvents: insights from molecular dynamics simulation studies. Biophys J 84, 1628-1641.

Stahl, M., Jeppsson-Wistrand, U., Mansson, M.O., and Mosbach, K.
(1991). Induced stereo- and substrate selectivity of bioimprinted $\alpha-$ chymotrypsin in anhydrous organic media. J Am Chem Soc 113, 9366-9368.

Tawaki, S., and Klibanov, A.M. (1992). Inversion of enzyme enantioselectivity mediated by the solvent. J Am Chem Soc 114, 1882-1884.

Van Der Spoel, D., Lindahl, E., Hess, B., Groenhof, G., Mark, A.E., and Berendsen, H.J. (2005). GROMACS: fast, flexible, and free. J Comput Chem 26, 1701-1718.

Vartanian, J.P., Henry, M., and Wain-Hobson, S. (2001). Simulating pseudogene evolution in vitro: determining the true number of mutations in a lineage. Proc Natl Acad Sci USA 98, 13172-13176.

Verma, M.L., Azmi, W., and Kanwar, S.S. (2008). Microbial lipases: at the interface of aqueous and non-aqueous media. A review. Acta Microbiol Immunol Hung 55, 265-294.

Wang, Q., Yang, G., Liu, Y., and Feng, Y. (2006). Discrimination of esterase and peptidase activities of acylaminoacyl peptidase from hyperthermophilic Aeropyrum pernix K1 by a single mutation. J Biol Chem 281, 18618-18625.

Wescott, C.R., and Klibanov, A.M. (1993). Solvent variation inverts substrate specificity of an enzyme. J Am Chem Soc 115, 1629-1631.

Yang, G., Bai, A., Gao, L., Zhang, Z., Zheng, B., and Feng, Y. (2009). Glu88 in the non-catalytic domain of acylpeptide hydrolase plays dual roles: charge neutralization for enzymatic activity and formation of salt bridge for thermodynamic stability. Biochim Biophys Acta 1794, 94-102.

Yang, L., Dordick, J.S., and Garde, S. (2004). Hydration of enzyme in nonaqueous media is consistent with solvent dependence of its activity. Biophys J 87, 812-821.

Zaks, A., and Klibanov, A.M. (1985). Enzyme-catalyzed processes in organic solvents. Proc Natl Acad Sci USA 82, 3192-3196.

Zheng, L., Baumann, U., and Reymond, J.L. (2004). An efficient onestep site-directed and site-saturation mutagenesis protocol. Nucleic Acids Res 32, e115. 